\title{
Caracterización de la Atención Médica: Caso Salud Preventiva "Iglesia del Nazareno" del Cantón Riobamba, Provincia De Chimborazo, 2018
}

Characterization of Health Care: Case Preventive Health of the "Church of the Nazarene" of the Canton Riobamba, Chimborazo Province, 2018

\section{Nelson Mauricio Moreno Moreira ${ }^{1}$, Paola Belén Salazar Montero², Evelyn Carolina Villarroel Ponce ${ }^{3}$, Sandy Yomary Riofrío García ${ }^{4}$ \& Adrián Paul Llerena Asadobay 5}

\begin{abstract}
. https://doi.org/10.32/cienciadigital.v3i1.947

In order for the Sustainable Development Goals proposed by WHO until the year 2030 to be met, the problem must be resolved from its structure in the field of health and well-being.

The purpose of this publication is to benefit from the retrospective to establish adequate health care practices that guarantee that it is of quality and in favor of patient improvement.
\end{abstract}

In the present medical attention, it is sought to do more promotion and prevention of health, to execute a self-evaluation, and to articulate the technical-administrative-

\footnotetext{
1 Médico General. Docente del Instituto Tecnológico Superior Riobamba. Riobamba - Ecuador. morenomauricio@live.com.gmoreno@intitutos.gob.ec

${ }^{2}$ Master en Prevención de Riegos Laborales. Médico General. Docente del Instituto Tecnológico Superior Riobamba. Riobamba - Ecuador. paosalazarm88@hotmail.com psalazar@intitutos.gob.ec

3 Médico General. Docente del Instituto Tecnológico Superior Riobamba. Riobamba - Ecuador. joflim98@hotmail.com evillarroel@intitutos.gob.ec

4 Médico General. Docente del Instituto Tecnológico Superior Riobamba. Riobamba - Ecuador. yomary_2008@gmail.com sriofrio@intitutos.gob.ec

${ }^{5}$ Médico General. Estudiante de Postgrado en Dirección en Gerencia Hospitalaria Universidad Internacional de la Rioja. Riobamba - Ecuador. adrianchuspr@ hotmail.com 
medical trilogy, in order to control the quality of the medical professional's attention, fundamentally in the which refers to a medical practice based on evidence, discipline, professional ethics, and the implementation of methods that promote greater efficiency and effectiveness in the provision of health services.

Medical care is described within the framework of national and international parameters developed by health entities such as: MSP, ODNA, WHO / PAHO, USDA, CDC. With a comprehensive and holistic approach to the pediatric patient, framed in the promotion and prevention of health, characteristics of primary health care.

The present medical care, in addition to being preventive and curative, characterizes the quality of medical care, to seek self-evaluation and articulate the technicaladministrative-medical trilogy, in order to seek strategies to improve the quality of health care.

Keywords: Characterization, Health Care, Health, Preventive.

\section{Resumen.}

Para que los Objetivos de Desarrollo Sostenible planteados por la OMS hasta el año 2030 se cumplan, se debe resolver la problemática desde su estructura en el ámbito de la salud y el bienestar.

El propósito de la presente publicación es beneficiarse de la retrospectiva para establecer adecuadas prácticas de atención médica que garanticen que ésta sea de calidad y en favor de la mejoría del paciente.

La atención médica se encuentra descrita en el marco de parámetros nacionales e internacionales desarrollados por entidades de salud como: MSP, ODNA, OMS/OPS, USDA, CDC. Con un enfoque integral y holístico del paciente en edad pediátrica, enmarcado en la promoción y prevención de la salud, características de la atención primaria de la salud.

La presente atención médica, busca además de ser preventiva y curativa, caracterizar la calidad de atención médica, para procurar la autoevaluación y articular la trilogía técnico-administrativo-médica, para de este modo buscar las estrategias para mejorar la calidad de la atención en salud.

Palabras claves: Caracterización, Atención Médica, Salud, Preventiva. 


\section{Introducción.}

Riobamba, la Sultana de los Andes, es una ciudad ubicada en la región sierra-centro, por lo cual es conocida también como el corazón del Ecuador. Ubicada en la provincia de Chimborazo, con la mayor cantidad de población indígena de todo el país. Encontramos asimismo una diversidad de situaciones socioeconómicas, con áreas muy desprotegidas, y de situaciones familiares decadentes producto del trabajo arduo de las cabezas de familia en el tema de la agricultura y la venta de productos de las cosechas en cada 'nueva luna'. Los escenarios de estas ferias son: La Merced, La Condamine, San Alfonso, Mercado Mayorista, Oriental, Santa Rosa, San Francisco, La Esperanza y Plaza Dávalos.

A pesar del considerable aumento que ha experimentado la ciudad tanto en extensión como en población, la ciudad de Riobamba está dividida en 5 parroquias urbanas: Maldonado, Veloz, Lizarzaburu, Velasco y Yaruquíes. Las cuatro parroquias nombradas en primer lugar fueron producto de la distribución de la ciudad a su llegada a la llanura de Tapi en 1797 y la parroquia de Yaruquíes fue incorporada como parroquia urbana en 1965, teniendo una separación que cada vez ha ido acortándose.

La iglesia del Nazareno localización del actual chequeo médico se encuentra ubicada en la parroquia Velasco al norte de la ciudad, en el By Pass salida a Quito, exactamente en el Barrio San Miguel de Tapi, que alguna vez sería parroquia rural por lo que conserva ciertas características de esas condiciones de ruralidad, incluso las de pobreza extrema, saneamiento precario, inseguridad, drogadicción, alcoholismo, y una serie de problemas, convirtiéndose en uno de los barrios más desprotegidos de la ciudad.

\section{Marco Teórico}

\section{Calidad de atención en Salud}

El concepto de "calidad" proviene del latín qualitis, conjunto de cualidades que constituyen la manera de ser de una persona o cosa, y es sinónimo de cualidad, clase, aptitud, excelencia, categoría, superioridad $^{\mathrm{i}}$

P. Crosby dice: "La calidad debe definirse como ajuste a las especificaciones y no como bondad o excelencia", y J. Juran define "calidad" como "aquellas características que responden a las necesidades del cliente y la ausencia de deficiencias"1

Calidad de atención es un conjunto de cosas, no solo es que esto sea bonito, esa calidad que le va a dar el médico, la atención, la calidez que le va a brindar, la confianza y tampoco solo es eso, sino que también contemos con todo lo necesario, con ambientes calentitos, con ambientes adecuados, con medicamentos a la mano, con todo eso, o sea es un conjunto de cosas que hacen la calidad (El Alto).

Según el personal sanitario, los impedimentos para ofrecer una buena calidad de atención no se resuelven mediante soluciones que estén a su alcance. Consideran que son las instancias 
superiores las que pueden y deben garantizar los cambios necesarios del sistema. La falta de insumos, ítems y personal cualificado son algunos de los elementos que destacan como obstáculos que impiden ejercer la profesión en todas sus dimensiones. Encontrándose en peor situación las zonas rurales que las ciudades. Por otro lado, hay que tener en cuenta que, a causa de las limitaciones resolutivas de los centros de salud de las zonas rurales, se derivan numerosos enfermos a las ciudades. En este contexto, son los hospitales de tercer nivel los principales receptores de la población con problemas de salud.

A los problemas mencionados se suman los relacionados con el funcionamiento de los centros de salud, como lo destaca el personal sanitario. En este caso, hacen referencia a la organización interna de los centros: organización de los recursos humanos; de los estudiantes; suministro de medicamentos e insumos; la ética profesional y el trato -entendido como calidad de atención- que se otorga a los pacientes. No como un elemento que afecta a los sujetos individuales sino a la organización interna de los servicios.

En Latinoamérica presenta diferentes modelos de prestación de servicios de salud, y en ese contexto es conveniente conocer las diferentes experiencias para alcanzar una visión general de las mismas, a fin de poder evaluarlas, y de ser posible, implantarlas en nuestro país con el ánimo de mejorar el sistema actual.

Argentina se distinguió durante muchos años por la eficiencia y calidad de su sistema de salud, pero hace ya largo tiempo que el área ha venido disminuyendo sus capacidades y enfrenta problemas cada vez más graves. Encontramos un sistema que se ha caracterizado por ser heterogéneo con respecto a la cobertura, las características de la población atendida y las fuentes de financiamiento. En 1997 se transfirieron los hospitales a las provincias y de éstas a los municipios. La política de reforma del sector de la salud pretende lograr la hegemonía del sector público sobre los otros, con plena vigencia del derecho a la salud, con requisitos de equidad, solidaridad, eficacia, eficiencia y calidad, cobertura a toda la población y descentralización.

En Brasil, a pesar de la promulgación de la Constitución de1988, que instituyó el Sistema Único de Salud (SUS), en el que se garantizan todos los principios de universalidad, equidad e integralidad de la atención, con principios operativos de descentralización política, administrativa, jerarquización y control social, hay, en realidad, tres tipos de financiamiento (seguros y planes privados, seguridad social y gobierno), lo que determina la desigualdad de acceso de la población a los diferentes servicios públicos y privados.

Chile tiene un sistema de salud mixto, compuesto de fondos y prestadores tanto públicos como privados, liderado por el Ministerio de Salud, el cual tiene como tarea el diseño de políticas y programas, la coordinación de las entidades del área, la supervisión, la evaluación y el control de las políticas de salud.

En Ecuador, el modelo de salud está soportado en el Aseguramiento Universal en Salud (AUS). Delimitado por dos grandes macropropuestas: Incrementar de un $20 \%$ al $100 \%$ la 
cobertura en salud y competencia médica, excelencia, integralidad y calidad de la atención en salud. El sistema de Aseguramiento Universal y Salud contempla prestar servicios de promoción, prevención y recuperación de la salud, tomando en cuenta las capacidades diferenciales de pago de las personas o familias, como aporte complementario al financiamiento. Sistemas Descentralizados de Salud y Modelos de Autonomía de Gestión de Servicio. Este sistema debería ser muy positivo para la población, aunque, en realidad, se encuentran algunos resultados negativos: Los avances registrados no reportan grandes cambios en los indicadores de salud y en el funcionamiento de los servicios, el acceso y la calidad de la atención se ha deteriorado debido a una baja tasa de crecimiento económico, problemas de tipo estructural que se han agravado por las crisis sociales y políticas. (Delgado1, 2010)

\section{Epidemiología de la población pediátrica en el contexto de Latinoamérica y el Ecuador}

En cuanto al perfil epidemiológico, el país está experimentando un giro de las causas de mortalidad hacia los problemas crónicos degenerativos. Los problemas nutricionales son en el país un problema de salud pública, tanto la desnutrición como el sobrepeso, que en los últimos años se han incrementado y tienen una clara relación con las condiciones de vida, el acceso a alimentos y patrones de consumo. En este sentido, la desnutrición crónica afecta fundamentalmente a niños y niñas indígenas, a la población que habita en zonas rurales donde hay mayores índices de pobreza.

Según la Organización de Naciones Unidas continúa realizándose ciertos progresos en la reducción del retraso del crecimiento, los niveles siguen siendo inaceptablemente elevados. Casi 151 millones de niños menores de cinco años de todo el mundo, o más del $22 \%$, estaban afectados por retraso del crecimiento en 2017. La emaciación continúa afectando a más de 51 millones de niños menores de cinco años del mundo y estos niños tienen un mayor riesgo de morbilidad y mortalidad.

A referencia por El Fondo de las Naciones Unidas para la Infancia (UNICEF) cerca de 200 millones de niños, aproximadamente un tercio de los niños menores de cinco años, sufren desnutrición crónica. Un 90\% vive en Asia y África. En estos dos continentes, las tasas en niños menores de cinco años son muy elevadas, con un $40 \%$ en Asia y un $36 \%$ en África. El $80 \%$ de los niños con desnutrición crónica vive en 24 países. De los 10 países que más contribuyen a la cifra mundial de desnutrición crónica entre los niños, seis de ellos se encuentran en Asia: Bangladesh, China, Filipinas, India, Indonesia y Pakistán. Todos estos países tienen poblaciones relativamente grandes. Por el contario un $13 \%$ de los niños menores de 5 años sufre desnutrición aguda moderada, y un 5\% desnutrición aguda grave. Un total de 32 países tienen un $10 \%$ o más de niños menores de 5 años con desnutrición aguda grave, que requieren medidas urgentes.

La Organización de las Naciones Unidas para la Alimentación y la Agricultura (FAO) y la Organización Panamericana de la Salud (OPS), por medio de la publicación "El Panorama de la seguridad alimentaria y nutricional en América Latina y el Caribe 2017" informa por 
primera vez los avances de los países de la Región hacia las metas de los Objetivos de Desarrollo Sostenible (ODS) estableciendo que existe subalimentación en América Latina y el Caribe misma que aumenta en el último período de medición. Después de varios años de estancamiento, en el año 2016, alrededor de 42,5 millones de personas no cuentan con la cantidad suficiente de alimentos para cubrir sus necesidades calóricas diarias, esto es, un aumento de 2,4 millones de personas, lo que significa un incremento de $6 \%$ de la población subalimentada en comparación con el año anterior.

Por el contrario, en el Ecuador disminuye 18 puntos la tasa de desnutrición en menores de cinco años en los últimos 20 años. En la década de los 80 era del 41\%, mientras que el 2011 bajo al 23\%, esto a según los manifestado en el informe del Observatorio de los Derechos de la Niñez y Adolescencia (ODNA), tras una investigación entre 1990 y 2011.

En detalle se refiere además que según la Primera Encuesta de la Niñez y Adolescencia de la sociedad civil (2010), dos de cada cinco ecuatorianos menores de 5 años no crecieron lo suficiente para su edad. En las últimas dos décadas el país hizo esfuerzos para reducir la desnutrición. En 1986, cuatro de cada 10 niños y niñas menores de 5 años sufrían de desnutrición crónica; 12 años más tarde la cifra había descendido a tres de cada 10, sin embargo, con la crisis económica de finales del siglo pasado su descenso se detuvo y sólo fue hasta el 2004 que esa cifra nuevamente cayó. Entre el 2000 y el 2004, la proporción de niños y niñas menores de 5 años que no crecieron lo suficiente para su edad se redujo de $31 \%$ a $26 \%$. Destacando que actualmente se evidencia una reducción del $30 \%$ de la desnutrición en los últimos 24 años.

\section{Metodologia.}

En este estudio se realizó consulta médica general con sus implicaciones: la pre-consulta, en la que se realizó la preparación y tomas de signos vitales de los pacientes; la consulta, en la que se pone en práctica las destrezas clínicas de cada médico, y la postconsulta en la que se desarrolla una orientación nutricional, de estilos de vida y prácticas saludables.

Este estudio es descriptivo situándose sobre una base de conocimientos más sólida que los exploratorios. En estos casos el problema científico ha alcanzado cierto nivel de claridad, pero aún se necesita información para poder llegar a establecer caminos que conduzcan al esclarecimiento de relaciones causales. En el área de la investigación clínica son habituales los estudios que describen la frecuencia de presentación de las enfermedades, y de los diferentes cuadros clínicos y los que examinan la asociación de características clínicas con signos radiológicos, histopatológicos, bioquímicos, etc. Para lo cual en el presente trabajo se han descrito cuáles son las enfermedades presentadas posterior al examen médico general, determinando de esta manera la frecuencia de su presentación y siendo además analítico ya que consiste en la desmembración de un todo, descomponiéndolo en sus partes o elementos para observar las causas, la naturaleza y los efectos. ${ }^{\mathrm{xi}}$ Describiendo entonces a esta investigación como analítica, ya que se realiza observación mediante el examen médico general, toma de medidas antropométricas, estableciendo el diagnostico nutricional mediante tablas y curvas de crecimiento utilizadas por la CDC, analizado los trastornos nutricionales, presentados en la población sujeto de estudio. 


\section{Actividades realizadas}

\section{Toma de medidas antropométricas y estado nutricional}

Se realizó sistemáticamente la toma de medidas antropométricas, las cuales son peso para la edad, talla para la edad, y el cálculo del índice de masa corporal (IMC) para la edad., para establecer el estado nutricional de los pacientes y tomar medidas correctivas en caso de que el caso lo amerite.

Los datos antropométricos se grafican finalmente en las tablas de curvas de crecimiento de la CDC, que nos permite en una misma hoja la colocación de todos los datos concernientes al estado nutricional, como la fecha, edad, talla, peso, IMC; además del gráfico de la talla y el peso en pacientes en edades comprendidas entre los 2 a 20 años, para hombre y para mujer según corresponda; se usaron también las curvas de la OMS para el gráfico de talla para la edad, peso para la edad e IMC, en niños menores de 5 años, que en las curvas de la CDC, no pueden ser graficadas debido a los rangos de edad.

El apartado de la curva del peso para la edad (P/E) arroja los siguientes estados nutricionales: peso muy elevado, peso elevado, peso normal, peso bajo, peso bajo severo, y los 'riesgos' de incurrir en uno u otro de los estados nutricionales, que se especifican en las respectivas historias.

Por otra parte, la curva destinada para el gráfico de la talla para la edad (T/E) determina los siguientes estados nutricionales: talla muy alta, talla alta, talla normal, talla baja, talla baja severa, y los 'riesgos' de incurrir en uno u otro estado nutricional.

El análisis de las curvas de índice de masa corporal, que corresponden al cálculo de la relación que guarda el peso para la talla de acuerdo a la edad, puede indicar cualquiera de los siguientes estados: emaciación o delgadez severa, emaciación o delgadez, resultado normal, sobrepeso y obesidad.

El conjunto de las medidas antropométricas $(\mathrm{P} / \mathrm{E}, \mathrm{T} / \mathrm{E}, \mathrm{IMC} / \mathrm{E})$, brindan las herramientas suficientes para establecer un diagnóstico nutricional, aupado con el examen médico general, investigación de comorbilidades, estilos de vida, y de la inferencia que pudiera tener la heredabilidad familiar en cuanto a patrones de crecimiento se refiere.

El abordaje del estado nutricional de los pacientes se lo hace en base a los lineamientos nutricionales de la Guía "Mi Plato", creada por el Departamento de Agricultura de EE.UU, para ayudar a los padres a alimentar a sus hijos con comidas nutritivas y equilibradas.

Este colorido plato está dividido en 5 partes, una para las verduras, otra para las frutas, otra para los cereales, otra para los alimentos con alto contenido de proteínas, y un espacio para los lácteos. Es una importante mejora con respecto a la confusa y compleja pirámide de la alimentación que antes utilizaba el USDA como guía nutricional. 


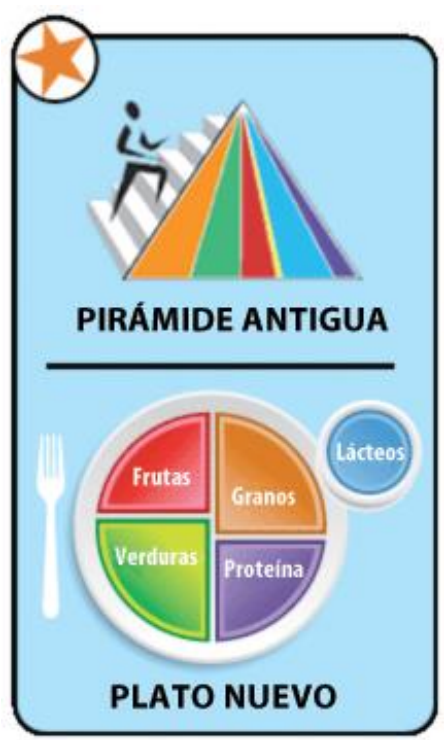

Imagen 1. Comparación de la pirámide antigua (poco didáctica), versus el nuevo plato que ofrece mensajes claros que los padres pueden entender fácilmente, como:

- Llenar la mitad del plato de su hijo con verduras y frutas.

- Hacer que, por lo menos, la mitad de los cereales que le sirva sean integrales, como avena o arroz integrales.

- Servir leche descremada o semidescremada (al 1\%) y agua en vez de bebidas azucaradas.

- Cuando se compre alimentos envasados o platos precocinados, elija los que tengan bajo contenido de sodio.

- No servir porciones demasiado grandes.

\section{Control médico general}

- Los niños representan el futuro, y su crecimiento y desarrollo saludable deben ser una de las máximas prioridades para todas las sociedades. Los niños y los recién nacidos en particular son especialmente vulnerables frente a la malnutrición y enfermedades infecciosas, que son prevenibles o tratables en su mayoría.

- El control médico general busca en la presente revisión busca realizar un enfoque holístico e integral acerca de lo que comprende el amplio concepto de la salud. La promoción y la prevención de la salud son la consigna de los chequeos médicos, cuyo 
desarrollo empieza en los datos de filiación y anamnesis, y culmina en el tratamiento, referencia, y/o seguimientos.

\section{Programa de desparasitación}

Según recomendaciones de la Organización Mundial de la Salud, para evitar las helmintiasis transmitidas por el suelo, que son enfermedades parasitarias causadas por diferentes especies de gusanos, se prescribió desparasitación rutinaria de toda la población atendida e incluso tutores. El antecedente es que los niños infectados por parásitos sufren deterioro físico, nutricional y cognitivo, por lo tanto, la desparasitación fue llevada a cabo en base a lo siguiente:

- Desparasitación periódica para eliminar los gusanos;

- Educación sanitaria para prevenir la reinfección; y

- Mejora del saneamiento para reducir la contaminación del suelo por huevos infectivos.

Siguiendo los lineamientos del órgano rector de la salud, se prescriben medicamentos seguros y eficaces para controlar la infección, en este caso poniendo en práctica los conocimientos costo-efectividad de los medicamentos que la OMS recomienda.

Citando textualmente un extracto tomado de la nota descriptiva presente en la página de la OMS, nos dice que:

"La estrategia de control de las helmintiasis transmitidas por el suelo consiste en controlar la morbilidad tratando periódicamente a las personas en situación de riesgo que viven en zonas endémicas. Las personas en riesgo son las siguientes:

- Niños en edad preescolar;

- Niños en edad escolar;

- Mujeres en edad fecunda (en particular las embarazadas durante el segundo y tercer trimestres de la gestación y las mujeres lactantes);

- Adultos con algunas ocupaciones de alto riesgo, como recolectores de té o mineros.

La OMS recomienda el tratamiento farmacológico (vermífugo) periódico sin diagnóstico individual previo para todas las personas en situación de riesgo que vivan en zonas endémicas. El tratamiento debe administrarse una vez al año si la prevalencia de referencia de helmintiasis transmitidas por el suelo en la comunidad supera el $20 \%$ y dos veces al año si la prevalencia supera el 50\%. Esta intervención reduce la morbilidad porque hace disminuir la carga de gusanos. Además: 
- La educación sobre salud e higiene reduce los casos de transmisión y reinfección porque fomenta la adopción de conductas saludables;

- También es importante que existan sistemas adecuados de saneamiento, pero ello no siempre es posible en entornos con pocos recursos".

Fuente de información: Chequeo médico e historias cínicas.

Población: Niños desde los 3 años de edad hasta los 19 años pertenecientes al CDN de la Iglesia del Nazareno RIOBAMBA - CHIMBORAZO. 2018.

Muestra: 147

\section{Resultados.}

La población total final atendible que constaba de 200 pacientes, presentó la heterogeneidad de patologías esperadas en un chequeo de rutina, con la excepcionalidad de casos exhibida en la tabla de patologías encontradas.

Sin embargo, es importante destacar el ausentismo que en este proyecto se encontró, aproximadamente el $12 \%$ (ver Tabla 1). Siendo la población final atendida de 176 pacientes $(88 \%)$.

Tabla 1. Población atendible y atendida en el CDN de la Iglesia del Nazareno RIOBAMBA-CHIMBORAZO 2018

\begin{tabular}{|r|r|c|}
\cline { 2 - 3 } \multicolumn{1}{c|}{} & \multicolumn{1}{|c|}{$\%$} & \\
\hline POBLACIÓN TOTAL & & 195 \\
\hline POBLACIÓN PARA ATEN & 100 & 155 \\
\hline AUSENTISMO & 5,2 & 8 \\
\hline POBLACIÓN ATENDIDA & 94,8 & $\mathbf{1 4 7}$ \\
\hline TOTAL HOMBRES & 157,82 & 232 \\
\hline TOTAL MUJERES & 168,03 & 247 \\
\hline
\end{tabular}

Realizado por: Autores

Gráfico 1. Población atendida clasificada por género en el CDN de la Iglesia del Nazareno RIOBAMBA - CHIMBORAZO. 2018 


\section{POBLACIÓN ATENDIDA}

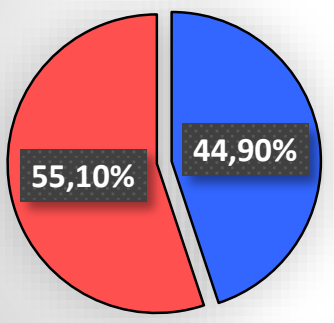

口TOTAL HOMBRES

$\square$ TOTAL MUJERES

La tabla 1 y gráfico 1 nos demuestran breves datos sobre la población atendida y las respectivas proporciones por género que en ésta suceden. Se deja entrevisto que la asistencia al programa de atención médica ha sido satisfactoria, solamente encontramos un $8 \%$ de ausentismo.

Tabla 2. Población por grupos etários atendida en el CDN de la Iglesia del Nazareno RIOBAMBA - CHIMBORAZO. 2018

\begin{tabular}{lcc}
\hline & POBLACION POR GRUPO ETARIO & \\
\hline$<5$ AÑNOS & 0 & $0 \%$ \\
5-9 AÑNOS & 83 & $56,46 \%$ \\
10-14 AÑ $O S$ & 36 & $24,48 \%$ \\
15-19 AÑ $O S$ & 28 & $19,04 \%$ \\
TOTAL & 147 & $100 \%$ \\
\hline
\end{tabular}

\section{Realizado por: Autores}

Gráfico 2. Porcentaje de la población por grupos etários atendida en el CDN de la Iglesia del Nazareno RIOBAMBA - CHIMBORAZO. 2018 


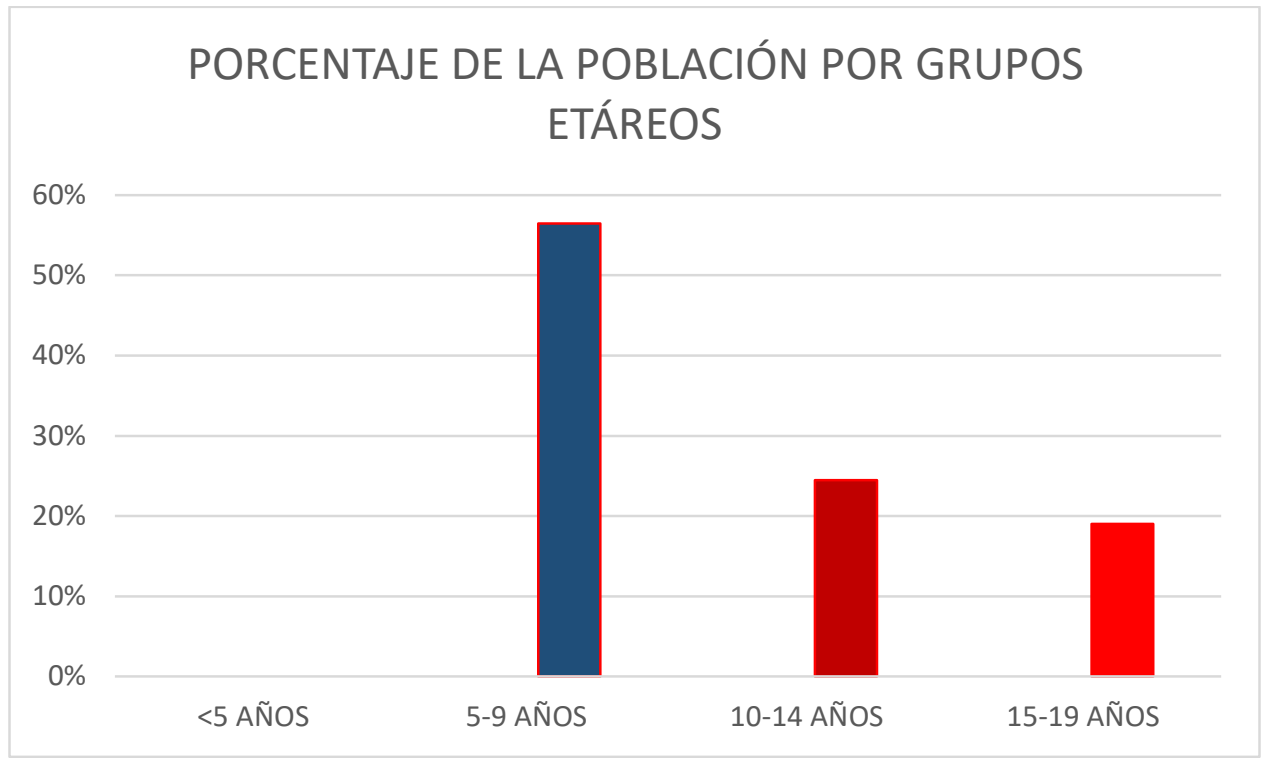

Realizado por: Autores

La edad, dentro de los datos de filiación, es un dato imprescindible para el médico, pues existe una evidente relación entre la mayoría de enfermedades y los diferentes periodos de la vida, los cuales, a su vez, imprimen a aquellas un curso evolutivo distinto, diferente terapéutica e incluso distinto pronóstico. En el Gráfico 2 se refleja la distribución poblacional atendida, por grupos de edad, observándose que la gran mayoría de niños (56\%), pertenecen a edades comprendidas entre los 5 y 9 años, el $24 \%$ son pacientes en edades comprendidas entre los 10 y 14 años, y el 19\% corresponde a adolescentes de 14 a 19 años. En el presente chequeo no se encontraron niños de edad preescolar, es decir menores de 5 años.

Tabla No. 3 Patologías presentadas CDN de la Iglesia del Nazareno RIOBAMBA CHIMBORAZO. 2018.

\begin{tabular}{ccc}
\hline PATOLOGÍAS & $\mathbf{N}^{\circ}$ DE & PORCENTAJE \\
\hline CASOS & $77,0 \%$ \\
\hline PARASITOSIS INTESTINAL (B82.9) & 114 & $25,0 \%$ \\
DESNUTRICIÓN CRÓNICA (E45.X) & 37 & $24,0 \%$ \\
PITIRIASIS ALBA (L30.5) & 36 & $14,0 \%$ \\
RINOFARINGITIS AGUDA (J00.X) & 21 & $12,0 \%$ \\
PEDICULOSIS CAPITIS (B85.0) & 18 & $11,0 \%$ \\
TALLA BAJA (R62) & 17 & $11,0 \%$ \\
SOPLO CARDIACO (R01) & 17 & $10,0 \%$ \\
CARIES (K02.9) & 16 &
\end{tabular}


Vol. 3, N².1, p. 42-59, abril - junio, 2019

SOBREPESO Y OBESIDAD (E66)

TRASTORNOS DE LA REFRACCIÓN NO ESPECIFICADOS

RINITIS ALÉRGICA (J30.4)

OTRAS

DESNUTRICIÓN AGUDA (E44.X)

FARINGITIS AGUDA (J02.9)

GASTRITIS AGUDA Y DISPEPSIA (K27)

LUMBAGO NO ESPECIFICADO (M54.5)

HIPERTROFIA DE AMÍGDALAS (J34.2)

DISMENORRA PRIMARIA

DEFORMIDAD TIPO VALGO

ASMA

INFECCIÓN DE VÍAS URINARIAS (N76)

VAGINITIS (N76)

VERRUGAS VÍRICAS (B07)

SÍNDROME RAYNAULD

ADENOPATIA LOCALIZADA

DESVIACION TABIQUE NASAL

ANEMIA APLÁSICA IDIOPÁTICA SEVERA

INFLUENZA TIPO B

CONJUNTIVITIS ALÉRGICA

CRISIS DE AUSENCIA (G40.7)

AMIGDALITIS AGUDA (J03.9)

\begin{tabular}{|c|c|}
\hline 14 & $9,0 \%$ \\
\hline 10 & $6,8 \%$ \\
\hline 10 & $6,8 \%$ \\
\hline 9 & $6,0 \%$ \\
\hline 6 & $4,0 \%$ \\
\hline 5 & $3,0 \%$ \\
\hline 3 & $2,0 \%$ \\
\hline 1 & $1,6 \%$ \\
\hline 2 & $1,3 \%$ \\
\hline 2 & $1,3 \%$ \\
\hline 2 & $1,3 \%$ \\
\hline 2 & $1,3 \%$ \\
\hline 1 & $0,6 \%$ \\
\hline 1 & $0,6 \%$ \\
\hline 1 & $0,6 \%$ \\
\hline 1 & $0,6 \%$ \\
\hline 1 & $0,6 \%$ \\
\hline 1 & $0,6 \%$ \\
\hline 1 & $0,6 \%$ \\
\hline 1 & $0,6 \%$ \\
\hline 1 & $0,6 \%$ \\
\hline 1 & $0,6 \%$ \\
\hline 0 & $0,0 \%$ \\
\hline
\end{tabular}

Realizado por: Autores

Gráfico 3. Porcentaje de patologías encontradas durante la atención en el CDN de la Iglesia del Nazareno RIOBAMBA - CHIMBORAZO. 2018. 


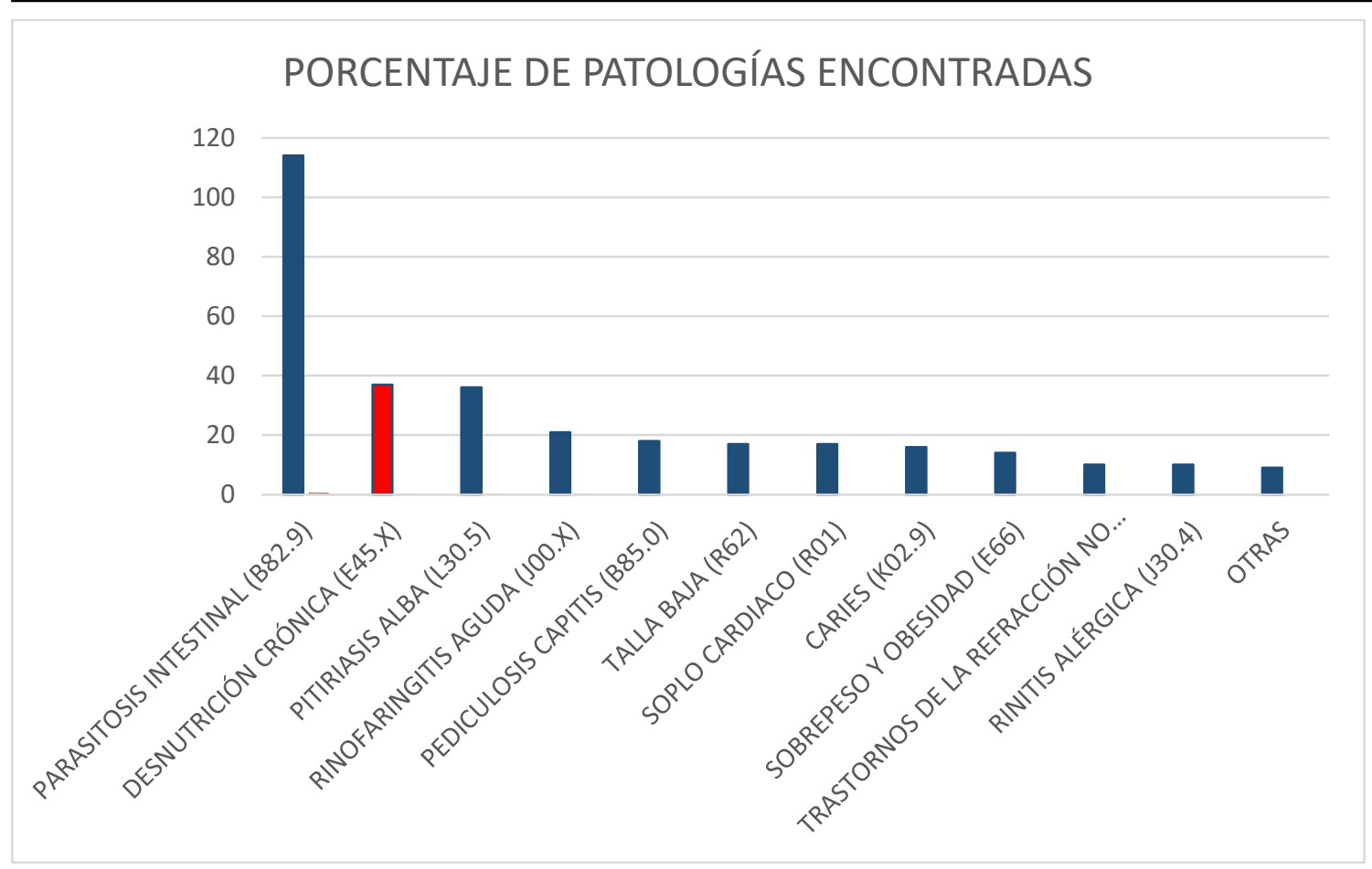

Realizado por: Autores

Las patologías presentadas en la tabla 3, son fiel reverbero del análisis situacional que se realiza en el siguiente apartado. Empero, la tabla en mención hace referencia de la incidencia de casos del total de la población atendida, ergo la suma de los puntos porcentuales no ponen en manifiesto el $100 \%$ de la población, ya que varios pacientes presentaban 2, 3,4, 5 e incluso 6 patologías al mismo tiempo, entonces analizamos la incidencia de las patologías, mas no de los niños enfermos, los cuales ya son analizados de manera particular en el informe individual.

En la tabla 3 y gráfico 3 se puede evidenciar que como las parasitosis bordean el $80 \%$ de la población, motivo más que suficiente para justificar la desparasitación masiva realizada a cerca del $100 \%$ de la población atendida.

A diferencia de otras poblaciones atendidas, en la población actual no despuntan los problemas odontogénicos. La desnutrición crónica en este caso $25 \%$ siendo el porcentaje más alto de todos los proyectos de atención médica por lo que se requiere especial atención para abordar este problema que tiene un gran componente socioeconómico. Por nuestra parte hemos otorgado la respectiva orientación nutricional y prescripción de antianorexiantes, multivitamínicos, e intervención de patologías subyacentes.

Tabla 4. Pacientes con necesidad de valoración por otras especialidades, CDN de la Iglesia del Nazareno RIOBAMBA - CHIMBORAZO. 2018. 


\begin{tabular}{lcc}
\hline POBLACIÓN ATENDIDA & $100.00 \%$ & 147 \\
\hline PACIENTES QUE REQUIEREN VALORACIÓN POR & $20.41 \%$ & 30 \\
OTRAS ESPECIALIDADES & $10.20 \%$ & 15 \\
ODONTOLOGÍA & $5,44 \%$ & 8 \\
OFTALMOLOGÍA/OPTOMETRISTA & $1,36 \%$ & 2 \\
PEDIATRÍA & $0,68 \%$ & 1 \\
DERMATOLOGÍA & $0,68 \%$ & 1 \\
NEUROLOGÍA & $0,68 \%$ & 1 \\
OTORRINOLARINGOLOGÍA & $0,68 \%$ & 1 \\
FISIOTERAPIA & $0,68 \%$ & 1 \\
HEMATOLOGÍA & $0,68 \%$ & 1 \\
CIRUGÍA VASCULAR & & \\
\hline
\end{tabular}

Realizado por: Autores

Gráfico 4. Pacientes con necesidad de valoración por otras especialidades, CDN de la Iglesia del Nazareno RIOBAMBA - CHIMBORAZO. 2018.

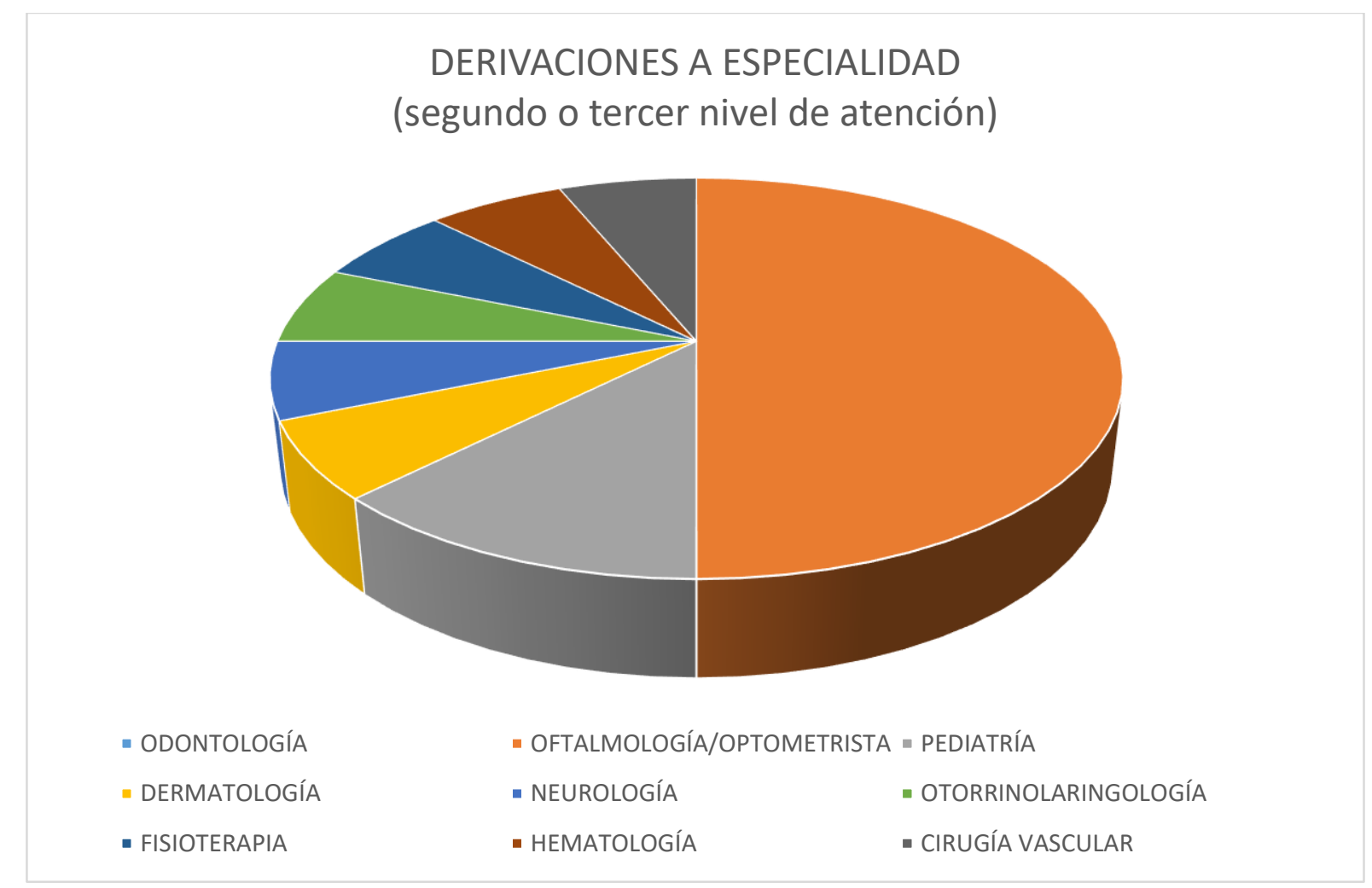

Realizado por: Autores 
Como es mandatorio en casi todos los chequeos la especialidad de la salud que más necesitamos para interconsulta es odontología, siendo el problema basal de los principales problemas de déficit nutricional.

En la presente atención la especialidad médica a la que más se ha solicitado derivación es a oftalmología, principalmente por los trastornos de refracción que provocan una diversidad de problemas como por ejemplo el mal rendimiento escolar, cefaleas crónicas, y fatiga.

\section{Análisis del contexto situacional}

$\mathrm{Si}$ analizamos los trastornos nutricionales solamente en un contexto aparte, encabezarían la lista de patologías encontradas con un $50 \%$ de la población. De éstos la mayor parte corresponde al déficit nutricional con $41 \%$. El porcentaje restante corresponde a problemas de obesidad y sobrepeso. (Ver Tabla 3).

La causa de morbilidad y el efecto de muchas otras morbilidades son los trastornos nutricionales, que merecen un análisis poco más que concienzudo. El enfoque preventivo de la atención, nos lleva a tomar medidas como la desparasitación, la orientación dietoterapéutica, la suplementación de micronutrientes y suplementos alimenticios, y la investigación de patologías subyacentes. Los déficits nutricionales, son causa de importante morbilidad, disminución en procesos de aprendizaje, en fin a que el sistema en un país se vea afectado desde las entrañas. Por lo tanto, se realzan consistentes actividades para revertir ciertos problemas nutricionales. La orientación nutricional se queda en la charla médicopaciente, de ahí, el verdadero trabajo empieza en los hogares, en el CDN, en las instituciones educativas, etc.

Los problemas nutricionales, conllevan obcecado seguimiento, puesto que déficits nutricionales, especialmente los crónicos se hacen dificultosos de revertir, conviene vigilar, y si es posible modificar los hábitos y estilos de vida en sentido integral.

\section{Conclusiones.}

- La atención médica descrita en la actual publicación, se encuentra enmarcada enparámetros nacionales e internacionales desarrollados por entidades de salud como: MSP, ODNA, OMS/OPS, USDA, CDC. Con una orientación integral y holística del paciente en edad pediátrica, enmarcado en la promoción y prevención de la salud, características de la atención primaria de la salud. Asimismo, la sucesión de los procedimientos como tal, están alineado con el Modelo de Atención Integral del Sistema Nacional de Salud, en la cual se destaca una atención individual, familiar, 
comunitaria, inclusiva, intercultural, con enfoque de género y de derechos, de calidad y calidez.

\section{Referencias bibliográficas.}

- Delgado1, A. E. (2010 ). Evaluación de la calidad de la atención en. Salud Uninorte, 154.

- Freire. (24 de Mayo de 2013). Montalvo, un paraíso turístico. La Hora, pág. 4.

- $\quad$ HITA, S. R. (2010). REPRESENTACIONES SOCIALES DEL PERSONAL. En S. R. HITA, CALIDAD DE LA ATENCION EN SALUD . BOLIVIA : OPS.

- FERREIRA , C., \& GARCÍA, K. (2010). Mujeres y Hombres del Ecuador en Cifras III. Ecuador: Editorial Ecuador.

- MSP, S. N. de G., \& Gobernanza. (2013). Manual del Modelo de Atención Integral de Salud - MAIS. (MSP, Ed.). Quito: MSP.

- CDC. Growth Charts. 2000. https://www.cdc.gov/growthcharts/cdc_charts.htm

- USDA. Guía nutricional Mi plato. http://kidshealth.org/es/parents/myplate-esp.html

- INEC. Encuesta de Condiciones de Vida (ECV) 2006

- OMS. Temas de salud: Salud del Niño. http://www.who.int/topics/child_health/es/

- KIDS HEALTH. General Health for parents, for kids and teens. http://kidshealth.org/en/parents/general/

- OMS. Promoción de la Salud. Promover la salud, promover el desarrollo sostenible. http://www.who.int/healthpromotion/es/

- OMS. Helmintiasis transmitidas por el suelo. Nota descriptiva. Enero 2017. http://www.who.int/mediacentre/factsheets/fs366/es/

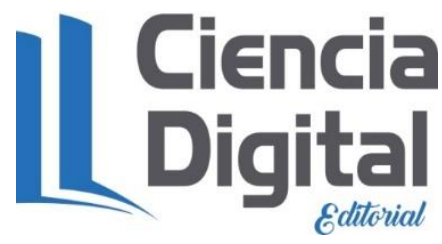


Para citar el artículo indexado.

Egas Moreno, F., Cifuentes, L., Toala Preciado, D., \& Vergara Cuadros, Y. (2019). Estudio para la implementación del manual de control interno caso (Gobierno Municipal del Cantón Puerto Quito, Provincia de Pichincha). Ciencia Digital, 9(2), 6-26.

https://doi.org/10.33262/cienciadigital.v9i2.372

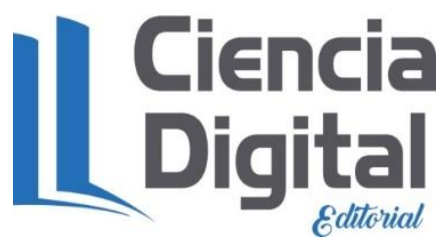

El artículo que se publica es de exclusiva responsabilidad de los autores y no necesariamente reflejan el pensamiento de la Revista Ciencia Digital.

El articulo queda en propiedad de la revista y, por tanto, su publicación parcial y/o total en otro medio tiene que ser autorizado por el director de la Revista Ciencia Digital.
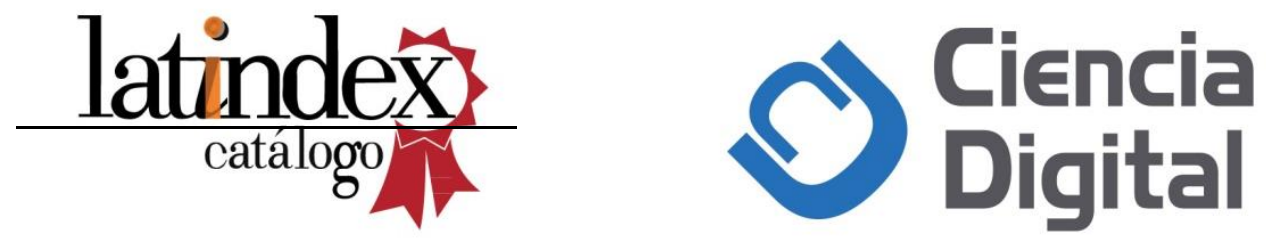